\title{
CONSTRUCTIVE EXISTENCE OF MINKOWSKI FUNCTIONALS
}

\author{
HAJIME ISHIHARA
}

(Communicated by Andreas R. Blass)

\begin{abstract}
In Bishop's constructive mathematics, the framework of this paper, there are many situations where we cannot easily prove the existence of functionals whose existence is a trivial consequence of classical logic. One such functional is the Minkowski functional of a convex absorbing set. We shall prove the existence of Minkowski functionals in various spaces, and apply the theorems to establish the locatedness of the kernel of linear mappings.
\end{abstract}

In Bishop's constructive mathematics, the framework of this paper, there are many situations where we cannot easily prove the existence of functionals whose existence is a trivial consequence of classical logic. One such functional is the Minkowski functional of a convex absorbing set.

We say that a convex subset $C$ of a normed linear space $X$ is absorbing if every $x$ in $X$ lies in $r C$ for some $r>0$ : note, for a convex absorbing subset $C$, that $0 \in C$ and that $0 \leq s<t$ implies $s C \subseteq t C$. The Minkowski functional $\mu$ of $C$ is defined by

$$
\mu(x) \equiv \inf \{r>0: x \in r C\} \quad(x \in X) .
$$

It is perhaps surprising that we cannot prove constructively that every convex absorbing subset in $\mathbf{R}$ has a Minkowski functional. For if we could do this, then, by considering a convex absorbing subset $C \equiv \bigcup_{n=1}^{\infty}\left(-1-a_{n}, 1+a_{n}\right)$ where $\left\{a_{n}\right\}$ is an increasing binary sequence, we would be able to prove the statement

$$
\exists n\left(a_{n}=1\right) \vee \forall n\left(a_{n}=0\right),
$$

which is called the limited principle of omniscience (LPO): in fact, if the Minkowski functional $\mu$ of $C$ exists, then either $2>\mu(2)$ or $\mu(2)>1$; in the former case, we have $2=r x$ for some $x \in C$ and $r$ with $0<r<2$; so there exists $N$ such that $x \in\left(-1-a_{N}, 1+a_{N}\right)$; if $a_{N}=0$, then $1<2 / r=x<1$, a contradiction; so $a_{N}=1$; in the latter case, if $a_{n}=1$ for some $n$, then $C=(-2,2)$; so $1 \geq \mu(2)>1$, a contradiction; so $a_{n}=0$ for all $n$. Constructive mathematicians do not accept LPO, and intuitionists and Russian constructive mathematicians can prove that LPO is false. For further comments on LPO, see [4, Chapter 1].

Received by the editors September 25, 1990 and, in revised form, February 6, 1991.

1980 Mathematics Subject Classification (1985 Revision). Primary 46R05; Secondary 03F65.

Key words and phrases. Minkowski functional, kernel of a linear mapping, constructive. 
We shall prove the existence of Minkowski functionals in a general normed linear space, a Banach space, and a finite-dimensional space. A crucial point in the existence theorems is the locatedness of a convex absorbing set: a subset $S$ of a metric space $X$ is said to be located in $X$ if

$$
d(x, S) \equiv \inf \{d(x, y): y \in S\}
$$

exists for each $x$ in $X$. Note that $C$ in the above example is not located: for if $C$ is located, then either $1>d(2, C)$ or $d(2, C)>0$; in the former case, we have $a_{n}=1$ for some $n$; in the latter case, $a_{n}=0$ for all $n$.

io end the paper, we use our main theorems to establish the locatedness of the kernel of linear mappings.

In the rest of the paper, we assume familiarity with the constructive theory of metric and normed spaces [1, Chapters 4 and 7]; we use $B_{X}(x, r)$ to denote the open ball with center $x$ and radius $r$ in a normed linear space $X$; and we apply the term Banach space to a complete normed linear space. (Unlike some authors, we do not require Banach spaces to be separable.)

We now turn to a lemma which plays a crucial role in the proofs of theorems.

Lemma 1. Let $C$ be a convex absorbing subset of a normed linear space $X$. Then $C$ has a Minkowski functional if and only if for all $x$ in $X$ and positive real numbers $s, t$ with $s<t$, either $x \notin s C$ or $x \in t C$.

Proof. Suppose that $\mu(x) \equiv \inf \{r>0: x \in r C\}$ exists for all $x \in X$. Then either $s<\mu(x)$ or $\mu(x)<t$. In the former case, if $x \in s C$ then we have $\mu(x) \leq s<\mu(x)$, and a contradiction; therefore $x \notin s C$. In the latter case, clearly $x \in t C$.

Conversely, suppose that for all positive real numbers $s, t$ with $s<t$, either $x \notin s C$ or $x \in t C$. Let $\alpha, \beta$ real numbers with $\alpha<\beta$, and set $s \equiv$ $(2 \max \{0, \alpha\}+\beta) / 3, t \equiv(\max \{0, \alpha\}+2 \beta) / 3$. Then either $\alpha<0$ or $0<\beta$; in the latter case, $s$ and $t$ are positive real numbers with $s<t$; so $\alpha<0$, or $x \notin s C$, or $x \in t C$. In the first two cases, $\alpha$ is a lower bound of the set $\{r>0 ; x \in r C\}$ : in fact, if $x \notin s C$ and $x \in r C$ for some positive real number $r$ with $r<s$, then we have $x \in s C$, a contradiction; therefore $s$ is a lower bound of the set $\{r>0: x \in r C\}$, and so is $\alpha$. In the last case, $t<\beta$. Thus, by [4, Chapter 2, Theorem 1.2], $\inf \{r>0: x \in r C\}$ exists.

Our first result is on the existence of a Minkowski functional in a general normed linear space.

Proposition 1. Let $C$ be a located convex absorbing subset of a normed linear space $X$ with nonempty interior. Then $C$ has a Minkowski functional.

Proof. Let $z \in C$ and let $\delta$ be a positive real number such that $B_{X}(z, \delta) \subseteq C$. Then there exists $r>0$ such that $-z \in r C$; so

$$
B_{X}(0, \delta)=B_{X}(z, \delta)-z \subseteq C+r C=(1+r) C,
$$

and therefore $B_{X}(0, \delta /(1+r)) \subseteq C$. Let $\delta^{\prime} \equiv \delta /(1+r)$, let $s, t$ be positive real numbers with $s<t$, and set $\varepsilon \equiv \delta^{\prime}(t-s)$. For each $x$ in $X$, either $d(x, s C)>0$ or $d(x, s C)<\varepsilon$. In the former case, $x \notin s C$. In the latter case, there exists $y \in s C$ such that $\|x-y\|<\varepsilon$, and hence we have

$$
x=y+(x-y) \in s C+\left(\varepsilon / \delta^{\prime}\right) C=t C .
$$


Thus $\inf \{r>0: x \in r C\}$ exists, by Lemma 1 .

We have seen in the example at the beginning of the paper that we cannot remove the locatedness of $C$ from the hypothesis of Proposition 1.

Note that for a convex absorbing subset $C$ with a Minkowski functional $\mu$, $C$ has nonempty interior if and only if $\mu$ is continuous: in fact, $B_{X}(0, \delta) \subseteq C$ if and only if $\mu(x)<1$ whenever $\|x\|<\delta$.

Classically, every closed convex absorbing subset of a Banach space has nonempty interior, by a version of Baire's theorem; but to prove it constructively, we need an assumption that the complement of the subset is located; see [4, Chapter 2, (2.5)]; so we need the assumption to show the existence of a Minkowski functional in a Banach space. The next result shows that we can prove the existence of the functional without the assumption, using the method in [3].

Theorem 1. Let $C$ be a closed located convex absorbing subset of a Banach space $X$. Then $C$ has a Minkowski functional.

Proof. Let $x \in X$, let $s, t$ be positive real numbers with $s<t$, and set $\varepsilon \equiv(t-s) / 2$. Then since $s C$ is complete and located, there exists $y \in s C$ such that if $x \neq y$, then $d(x, s C)>0$ by [4, Lemma (3.3)]. Construct an increasing binary sequence $\left\{\lambda_{n}\right\}$ such that

$$
\begin{aligned}
& \lambda_{n}=0 \Rightarrow\|x-y\|<1 /(n+1)^{2}, \\
& \lambda_{n}=1 \Rightarrow x \neq y .
\end{aligned}
$$

We may assume that $\lambda_{1}=0$. Define sequences $\left\{z_{n}\right\}$ in $X$ and $\left\{\xi_{n}\right\}$ in $\mathbf{R}$ as follows: if $\lambda_{n}=0$, set $z_{n} \equiv 0$ and $\xi_{n} \equiv 0$; if $\lambda_{n}=1-\lambda_{n-1}$, set $z_{k} \equiv n(x-y)$ and $\xi_{k} \equiv 1 / n$ for all $k \geq n$. Then $\left\{z_{n}\right\}$ and $\left\{\xi_{n}\right\}$ are Cauchy sequences in $X$ and $\mathbf{R}$, respectively: in fact, $\left\|z_{m}-z_{n}\right\| \leq 1 / n$ and $\left|\xi_{m}-\xi_{n}\right| \leq 1 / n$ whenever $m \geq n$. As $X$ and $\mathbf{R}$ are complete, there exist $z \in X$ and $\xi \in \mathbf{R}$ such that $z_{n} \rightarrow z$ and $\xi_{n} \rightarrow \xi$ as $n \rightarrow \infty$. We shall prove that $d(x-\xi z, s C)=0$. To this end, assume that $d(x-\xi z, s C)>0$. If $\lambda_{n}=1-\lambda_{n-1}$, then $\xi=1 / n$ and $z=n(x-y)$; so $x-\xi z=y \in s C$, a contradiction. Thus $\lambda_{n}=0$ for all $n$, so that $x-\xi z=x=y \in s C$; this contradiction ensures that $d(x-\xi z, s C)=0$. Let $r$ be a positive real number such that $z \in r C$. Either $\varepsilon<\xi r$ or $\xi r<2 \varepsilon$. In the former case, choose a positive integer $N$ such that $1 / N<\varepsilon / r$, and suppose that $\lambda_{n}=0$ for all $n \leq N$. If $\lambda_{n}=1-\lambda_{n-1}$ for some $n>N$, then $1 / N<\varepsilon / r<\xi=1 / n$, a contradiction. Thus $\lambda_{n}=0$ for all $n$, so that $0<\varepsilon / r<\xi=0$; this contradiction ensures that $\lambda_{n}=1$ for some $n \leq N$; therefore $d(x, s C)>0$; so $x \notin s C$. In the latter case, we have $d(x, s C+\xi z)=d(x,(s+\xi r) C)=0$. As $(s+\xi r) C$ is closed, we obtain

$$
x \in(s+\xi r) C \subseteq(s+2 \varepsilon) C=t C .
$$

So $\inf \{r>0: x \in r C\}$ exists, by Lemma 1 .

Our last theorem establishes a criterion for the existence of a Minkowski functional in a finite-dimensional space.

Theorem 2. Let $C$ be a convex absorbing subset of a finite-dimensional normed linear space $X$. Then $C$ has a Minkowski functional if and only if $C$ is located in $X$. 
Proof. It is easy to see that the interior of $C$ contains 0 ; see [2, Lemma 3]. So, if $C$ is located, then $C$ has a Minkowski functional by Proposition 1 .

Conversely, suppose that $\mu(x) \equiv \inf \{r>0: x \in r C\}$ exists for all $x \in X$. Let $\alpha, \beta$ be real numbers with $\alpha<\beta$. Then either $\alpha<0$ or $0<\beta$; in the latter case, since $\gamma \equiv \max \{0, \alpha\}<\beta$, either $\|x\|<\beta$ or $(\gamma+\beta) / 2<\|x\|$; so $\alpha<0$, or $\|x\|<\beta$, or $(\gamma+\beta) / 2<\|x\|$. In the first case, $\alpha$ is a lower bound of the set $\{\|x-y\|: y \in C\}$; in the second case, $0 \in C$ and $\|x-0\|<\beta$. In the last case, let $\varepsilon \equiv(\beta-\gamma) /(2\|x\|-(\gamma+\beta))$. Then since the map $x \mapsto \mu(x)$ is continuous, $d \equiv \inf \left\{\mu(y): y \in B_{X}(x, \gamma)\right\}$ exists; therefore either $d<1+\varepsilon$ or $d>1+\varepsilon / 2$. In the former case, there exists $y \in B_{X}(x, \gamma)$ such that $\mu(y)<1+\varepsilon ;$ hence, $y /(1+\varepsilon) \in C$ and

$$
\begin{aligned}
\left\|\frac{x-y}{1+\varepsilon}\right\| & =\frac{\|x+\varepsilon x-y\|}{1+\varepsilon} \leq \frac{\|x-y\|+\varepsilon\|x\|}{1+\varepsilon} \leq \frac{\gamma+\varepsilon\|x\|}{1+\varepsilon} \\
& =\frac{\gamma+(\beta-\gamma)\|x\| /(2\|x\|-(\gamma+\beta))}{1+(\beta-\gamma) /(2\|x\|-(\gamma+\beta))} \\
& =\frac{2 \gamma\|x\|-\gamma(\gamma+\beta)+(\beta-\gamma)\|x\|}{2\|x\|-(\gamma+\beta)+(\beta-\gamma)}=\frac{\gamma+\beta}{2}<\beta .
\end{aligned}
$$

In the latter case, if $\|x-y\|<\gamma$ for some $y \in C$, then $1+\varepsilon / 2<d \leq \mu(y) \leq 1$; this contradiction ensures that $\alpha \leq \gamma \leq\|x-y\|$ for all $y \in C$. So $C$ is located by $[4$, Theorem (1.2)].

The following example shows that we cannot expect a similar criterion in a infinite-dimensional space.

Brouwerian Example 1. A closed convex absorbing subset of a Hilbert space with nonempty interior such that it has a Minkowski functional but it is not located.

Let $l^{2}$ be the Hilbert space of square-summable sequences of real numbers with standard basis $e_{1}, e_{2}, \ldots$, and let $\left\{a_{n}\right\}$ be a binary sequence containing at most one 1 , and such that $a_{1}=0$. Define a bounded operator on $l^{2}$ by

$$
u\left(\sum_{n=1}^{\infty} x_{n} e_{n}\right) \equiv \sum_{n=1}^{\infty} x_{n}\left(e_{n}+2 a_{n} e_{1}\right)
$$

Then $u$ has a bounded inverse

$$
u^{-1}\left(\sum_{n=1}^{\infty} x_{n} e_{n}\right) \equiv \sum_{n=1}^{\infty} x_{n}\left(e_{n}-2 a_{n} e_{1}\right) .
$$

Clearly $C \equiv u\left(B_{l^{2}}(0,1)\right)$ is closed, convex, has nonempty interior, and has $\mu(x)=\left\|u^{-1}(x)\right\|$ as a Minkowski functional. Suppose that $C$ is located. Then either $d\left(2 e_{1}, C\right)>2 / \sqrt{5}$ or $d\left(2 e_{1}, C\right)<1$. In the first case, $\forall n\left(a_{n}=0\right)$; for if $a_{n}=1$, then $4\left(e_{n}+2 e_{1}\right) / 5 \in C$; therefore

$$
\frac{2}{\sqrt{5}}<d\left(2 e_{1}, C\right) \leq\left\|2 e_{1}-\frac{4\left(e_{n}+2 e_{1}\right)}{5}\right\|=\left\|\frac{2}{5} e_{1}-\frac{4}{5} e_{n}\right\|=\frac{2}{\sqrt{5}},
$$


a contradiction. In the second case, there exists $x \equiv \sum_{n=1}^{\infty} x_{n} e_{n}$ such that $\|x\|<1$ and $\left\|2 e_{1}-u(x)\right\|<1$; so

$$
\begin{aligned}
1 & >\left\|2 e_{1}-\sum_{n=1}^{\infty} x_{n}\left(e_{n}+2 a_{n} e_{1}\right)\right\| \geq\left\|\left(2-x_{1}-2 \sum_{n=1}^{\infty} x_{n} a_{n}\right) e_{1}\right\| \\
& \geq 2-\left|x_{1}\right|-2\left|\sum_{n=1}^{\infty} x_{n} a_{n}\right|>1-2 \sum_{n=1}^{\infty}\left|x_{n} a_{n}\right|,
\end{aligned}
$$

and therefore $\sum_{n=1}^{\infty}\left|x_{n} a_{n}\right|>0$. Hence $\exists n\left(a_{n}=1\right)$.

We shall apply the results which we have proved to show the locatedness of the kernel of linear mappings onto various spaces. To this end, we need the following proposition. Although the proposition differs from [4, Proposition (5.2)] in that the latter is stated for bounded maps, the proof of [4, Proposition (5.2)] does not use the fact.

Proposition 2. Let $T$ be a linear mapping of a normed linear space $X$ onto a normed linear space $Y$. Then $\operatorname{ker}(T)$ is located in $X$ if and ony if $T\left(B_{X}(0,1)\right)$ has a Minkowski functional.

Corollary 1. Let $T$ be an open linear mapping $T$ of a normed linear space $X$ onto a normed linear space $Y$ such that $T\left(B_{X}(0,1)\right)$ is located in $Y$. Then $\operatorname{ker}(T)$ is located in $X$.

Proof. Since $T\left(B_{X}(0,1)\right)$ is a located convex absorbing subset of $Y$ with nonempty interior, $T\left(B_{X}(0,1)\right)$ has a Minkowski functional, by Proposition 1. Hence $\operatorname{ker}(T)$ is located, by Proposition 2 .

We say that a linear mapping $T$ between normed linear spaces $X$ and $Y$ is sequentially open if, whenever $T x_{n} \rightarrow 0$ as $n \rightarrow \infty$, there exists a sequence $\left\{y_{n}\right\}$ in $\operatorname{ker}(T)$ such that $x_{n}+y_{n} \rightarrow 0$ as $n \rightarrow \infty$.

Corollary 2. Let $T$ be a sequentially open linear mapping $T$ of a normed linear space $X$ onto a Banach space $Y$ such that $T\left(B_{X}(0,1)\right)$ is located in $Y$. Then $\operatorname{ker}(T)$ is located in $X$.

Proof. By Theorem 1, $\bar{\mu}(T x) \equiv \inf \left\{r>0: T x \in r \overline{T\left(B_{X}(0,1)\right)}\right\}$ exists for all $x \in X$. Clearly, $\bar{\mu}(T x)$ is a lower bound of the set $\{r>0: T x \in$ $\left.r T\left(B_{X}(0,1)\right)\right\}$. Let $\varepsilon>0$. Then there exists a positive real number $r$ such that $r<\bar{\mu}(T x)+\varepsilon / 2$ and $T x \in r \overline{T\left(B_{X}(0,1)\right)}$; therefore there exists a sequence $\left\{x_{n}\right\}$ in $B_{X}(0,1)$ such that $r T x_{n} \rightarrow T x$. Since $T$ is sequentially open, we can find a sequence $\left\{y_{n}\right\}$ in $\operatorname{ker}(T)$ such that $r x_{n}+y_{n} \rightarrow x$. Choose a positive integer $N$ such that $\left\|x-\left(r x_{N}+y_{N}\right)\right\|<\varepsilon / 2$. Then $\left\|x-y_{N}\right\| \leq\left\|r x_{N}\right\|+$ $\left\|x-\left(r x_{N}+y_{N}\right)\right\|<r+\varepsilon / 2$; therefore $T x=T\left(x-y_{N}\right) \in(r+\varepsilon / 2) T\left(B_{x}(0,1)\right)$ and $r+\varepsilon / 2<\bar{\mu}(T x)+\varepsilon$. Hence $\mu(T x) \equiv \inf \left\{r>0: T x \in r T\left(B_{X}(0,1)\right)\right\}$ exists and equals $\bar{\mu}(T x)$.

The following corollary follows from Proposition 2 and Theorem 2. Since every bounded located subset of a finite dimensional space is totally bounded, the corollary is a generalization of [2, Theorem 1].

Corollary 3. Let $u$ be a linear mapping of a normed linear space $X$ onto a finite-dimensional normed linear space $Y$. Then $\operatorname{ker}(u)$ is located in $X$ if and only if $u\left(B_{X}(0,1)\right)$ is located in $Y$. 


\section{ACKNOWLEDGMENT}

The author would like to thank the referee for many useful comments and suggestions.

\section{REFERENCES}

1. E. Bishop and D. Bridges, Constructive analysis, Grundlehren Math. Wiss., no. 279, Springer-Verlag, Berlin, 1985.

2. D. Bridges, A. Calder, W. Julian, R. Mines, and F. Richman, Bounded linear mappings of finite rank, J. Funct. Anal. 43 (1981), 143-148.

3. D. Bridges and H. Ishihara, Linear mappings are fairly well-behaved, Arch. Math. 54 (1990), 558-562.

4. D. Bridges and F. Richman, Varieties of constructive mathematics, London Math. Soc. Lecture Note Ser., vol. 97, Cambridge Univ. Press, Cambridge and New York, 1987.

Faculty of INTEgrated Arts and SCIences, Hiroshima University, Hiroshima 730, Japan 